\title{
FRAGILIDAD Y ÚLCERAS POR PRESIÓN EN PERSONAS MAYORES INSTITUCIONALIZADAS
}

\author{
Mirian Santamaría-Peláez \\ Universidad de Burgos \\ mspelaez@ubu.es \\ Jerónimo González-Bernal \\ Universidad de Burgos \\ jejavier@ubu.es \\ Josefa González-Santos \\ Universidad de Burgos \\ mjgonzalez@ubu.es \\ Raúl Soto-Cámara \\ Universidad de Burgos \\ rscamara@ubu.es
}

Recepción Artículo: 7 septiembre 2020

Admisión Evaluación: 10 octubre 2020

Informe Evaluador 1: 18 octubre 2020

Informe Evaluador 2: 21 octubre 2020

Aprobación Publicación: 27 noviembre 2020

\section{RESUMEN}

Investigación transversal y de tipo descriptivo para estudiar la relación entre la situación de fragilidad de las personas mayores institucionalizadas y la presencia de úlceras por presión y el riesgo de sufrirlas; en una muestra de 194 participantes.

La presencia de úlceras por presión se recoge a través de un registro ad hoc, el riesgo de padecer úlceras por presión se mide con la escala de Norton y la fragilidad se con la herramienta Short Physical Performance Battery; ambos instrumentos con buenas propiedades psicométricas.

Los resultados no muestran relación significativa entre los grupos de fragilidad y la presencia de UPP, aunque resulta destacable la no existencia de ninguna persona con úlceras por presión en los grupos de personas robustas y prefrágiles; así, todas las personas que presentan úlceras por presión se catalogan como frágiles (21,4\%) o dependientes (78,6\%).

Sin embargo, sí que existen diferencias significativas entre los grupos de fragilidad que establece el Short Physical Performance Battery y el riesgo de úlceras por presión entre todos los grupos de fragilidad $(, 060>p>, 001)$ excepto en la relación prefrágil-robusto. Además, aparece una correlación positiva entre el riesgo de úlceras por presión y la situación de fragilidad, de forma que a mayor riesgo de UPP, existe mayor situación de fragilidad y/o dependencia. 


\section{FRAGILIDAD Y ÚLCERAS POR PRESIÓN EN PERSONAS MAYORES INSTITUCIONALIZADAS}

Los resultados obtenidos permiten la detección de la población con mayor riesgo de úlceras por presión, lo que posibilitaría implementar intervenciones precoces encaminadas a la prevención de las mismas en base a las diferencias encontradas entre los grupos.

Palabras clave: fragilidad; síndrome de fragilidad; úlceras por presión; institucionalización

\section{ABSTRACT}

Frailty and pressure ulcers in institutionalized older adults. Cross-cutting and descriptive research to study the relationship between the situation of frailty among institutionalized older people and the presence of pressure ulcers and the risk of them; in a sample of 194 participants.

The presence of pressure ulcers is collected through an ad hoc register, the risk of pressure ulcers is measured with the Norton scale and frailty is measured with the Short Physical Performance Battery tool; both instruments with good psychometric properties.

The results show no significant relationship between frailty groups and the presence of pressure ulcers, although it is noteworthy that no person with pressure ulcers exists in robust and prefrail groups; thus, all people with pressure ulcers are classified as frail (21.4\%) or dependents (78.6\%).

However, there are significant differences between the frailty groups established by the Short Physical Performance Batrery and the risk of pressure ulcers among all frailty groups $(, 060>p>, 001)$ except in the prefrail-robust relationship. In addition, a positive correlation appears between the risk of pressure ulcers and the situation of frailty, so that at higher risk of pressure ulcers, there is a greater situation of frailty and/or dependence.

Results achieved allow the detection of the population most at risk of pressure ulcers, which would make it possible to implement early interventions aimed at preventing them based on the differences found between the groups.

Keywords: Frailty; frailty syndrome; pressure ulcers; institutionalization

\section{INTRODUCCIÓN}

La fragilidad se considera como una de las tipologías en las que se podría clasificar a las personas mayores; estas son: mayores sanos, mayores con enfermedad crónica pero sin problemas funcionales, mayores frágiles en riesgo de sufrir episodios adversos, y mayores en situación de dependencia y en el final de la vida.

La fragilidad se considera un síndrome geriátrico debido a que cumple los criterios de los mismos; está íntimamente relacionada con el proceso de envejecimiento, no se trata de una enfermedad concreta, tiene una alta prevalencia, implica a varios órganos y sistemas del cuerpo, es multidimensional y se asocia a graves eventos adversos de salud (Abizanda y Rodríguez, 2014).

La situación previa a la discapacidad o la dependencia es un buen indicador del estado de salud y buen predictor de la misma. El concepto de fragilidad se aproxima a la valoración de este estado previo, de manera que se propone un método para detectarla y a partir de ahí se convierte en susceptible de intervención, por lo que podrá ser revertida (Ministerio de Sanidad, Servicios Sociales e Igualdad, 2014).

Aunque no existe una definición estandarizada, una mayoría define la fragilidad como un síndrome clínico prevalente en ancianos, que conlleva una disminución de la reserva fisiológica, lo que se conoce como estado de reducción de la homeostasis, y un desequilibrio multisistémico. Linda Fried señala cinco criterios incluidos en el fenotipo de fragilidad:

- Pérdida de peso involuntaria por malnutrición (4,5 kg en el último año).

- Sensación de fatiga y/o agotamiento.

- Disminución de la velocidad de marcha (menos de $0,8 \mathrm{~m} / \mathrm{s}$ ).

- Debilidad (medida por la fuerza de aprehensión).

- Reducción de la actividad física.

El diagnóstico de fragilidad se basa en la presencia de tres o más criterios, mientras que la condición de prefragilidad incluye uno o dos de ellos (Fried y cols., 2001). 
Las úlceras por presión (UPP) constituyen un importante problema de salud y la prevención es el método más eficiente para su abordaje; por lo tanto, la valoración del riesgo es clave en dicha prevención.

Mediante esta valoración del riesgo, se pretende identificar a aquellas personas que requieren de prevención, además de identificar aquellos factores específicos que suponen esa situación de riesgo.

Las UPP tienen en España un gran impacto epidemiológico, con una prevalencia en el año 2005 del 8,91 \% en hospitales, del 9,11\% en centros de atención primaria y del $13 \%$ en residencias geriátricas, y con una importante mortalidad (García, Pancorbo, Soldevilla y Blasco, 2008).

\section{OBJETIVOS DE LA INVESTIGACIÓN}

Comprobar si existe relación entre la existencia de UPP y la situación de fragilidad.

Comprobar si existe relación entre el riesgo de sufrir UPP y la situación de fragilidad.

\section{PARTICIPANTES}

194 personas mayores institucionalizadas en 4 residencias componen la muestra; dos en la provincia de Burgos, una en la provincia de Salamanca y otra en la provincia de Madrid; todas ellas a gestionadas por el mismo grupo empresarial, con el cual se firmó un acuerdo de confidencialidad específico para este estudio.

\section{MÉTODO}

Este estudio se centra en la manifestación de las variables en contextos naturales por lo que no pretende establecer una relación de causalidad entre ellas y es, por tanto, un estudio no experimental de tipo descriptivo que pretende explicar si existe o no relación entre las variables sin mediar manipulación de las mismas.

La toma de datos se llevó a cabo en los propios centros residenciales a cargo de su propio personal y se estableció un procedimiento de anonimización de los datos para garantizar la confidencialidad de los mismos. El procedimiento de muestreo y análisis de datos fue aprobado por el Comité de Ética de la Universidad de Burgos (IR 11/2018). Este estudio no conlleva ningún tipo de intervención, por lo que no resultará lesivo para ninguno de los participantes.

Las variables que conforman este estudio son:

- Fragilidad: medida con la Short Physical Performance Battery (SPPB).

- Variable cualitativa politómica: Adopta valores entre 0 y 12 divididos en: 0-3 dependiente; 4-6 frágil; 7-9 prefrágil; 10-12 robusto (Proyecto ViviFrail).

- Variable cuantitativa: adopta valor numérico entre 0 y 12, siendo puntuaciones por debajo de 10 indicativas de fragilidad (Ministerio de Sanidad, Servicios Sociales e Igualdad, 2014).

- Existencia o no de UPP: variable cualitativa dicotómica que adopta los valores si/no.

- Riesgo de caídas: Variable cuantitativa, medida con la Escala de J. H. Downton, que tomará valores entre 0 y 11.

El Documento consenso sobre fragilidad y caídas en personas mayores propone como primera opción para valorar la existencia o no de fragilidad la escala SPPB. Se trata de una prueba de ejecución ampliamente utilizada, sencilla y rápida (Ministerio de Sanidad, Servicios Sociales e Igualdad, 2014).

Está validada y ajustada por edad, sexo y comorbilidad; por lo que se trata de una herramienta fiable para la detección de la fragilidad y la predicción de la discapacidad (Cabrero, Cabañero y Ramos, 2012; Poveda, 2014; Poveda, 2015; Pavasini y cols, 2016; Gómez, Curcio, Alvarado, Zunzunegui y Guralnik, 2013).

Consta de tres pruebas diferenciadas: equilibrio, velocidad de la marcha y levantarse y sentarse en una silla cinco veces; cada una de ellas puntúa entre 0 y 4 y el total oscila entre 0 y 12.

La escala de Norton fue la primera escala de valoración del riesgo de desarrollar una UPP descrita en la literatura en 1962, desarrollada por Norton junto con McLaren y Exton-Smith, en una población de pacientes geriátricos. Desde entonces, ha sido difundida y utilizada ampliamente a nivel mundial (García, Pancorbo, Soldevilla y Blasco, 2008). 


\section{FRAGILIDAD Y ÚLCERAS POR PRESIÓN EN PERSONAS MAYORES INSTITUCIONALIZADAS}

Contiene cinco parámetros: estado mental; incontinencia; movilidad; actividad y estado físico. Cada uno de los parámetros puede tomar valores entre 1 y 4; así, la puntuación máxima será de 20 y la mínima será de 5.

Puntúa de forma negativa, es decir, una menor puntuación indica mayor riesgo. En la versión original el punto de corte se situó en 14 puntos, aunque posteriormente, en 1987, Norton lo modificó para situarlo en 16 para los casos de riesgo moderado y en 12 menos para los casos que presentan riesgo alto.

Algunos autores han sugerido que, además de ser útil en la detección de pacientes en riesgo de desarrollar úlceras por presión, puede ser útil también como método de detección de ancianos de riesgo en un hospital (Miralles y Esperanza, 2006).

La escala Norton, que había demostrado su utilidad, fue modificada ya que había varios autores que consideraban que la original generaba falsos negativos, especialmente en pacientes con alto riesgo de padecer UPP y que no se identificaban (Romanos y Casanova, 2017). Romanos y Casanova compararon la escala Norton modificada con la escala Braden, concluyendo que la escala Norton modificada cuenta con una sensibilidad del $67,91 \%(62,42-73,39)$ y con una especificidad del 78,66 \% (77,41-79,92) (González y cols., 2014).

\section{RESULTADOS}

Las pruebas que se realizan son no paramétricas ya que la prueba de Kolmogorof-Smirnov es significativa (ps.05).

Se han recogido datos sobre la existencia o no de UPP en 194 casos, de los cuales 180 no presentan UPP y 14 casos sí. Todos los casos de personas con UPP se encuentran dentro de los grupos de personas dependientes $(78,6 \%)$ y de personas frágiles $(21,4 \%)$; por el contrario, las personas prefrágiles y robustas no presentan UPP (tabla 1). 
Tabla 1. Grupos de fragilidad-presencia de UPP

\begin{tabular}{llccr}
\hline & & \multicolumn{2}{c}{ UPP } & Total \\
\cline { 3 - 4 } & & Sí & No & \\
\hline \multirow{2}{*}{ Dependiente } & Recuento & 11 & 80 & 91 \\
& Recuento esperado & 6,6 & 84,4 & 91,0 \\
& \% dentro de UPP & $78,6 \%$ & $44,4 \%$ & $46,9 \%$ \\
& Residuo corregido & 2,5 & $-2,5$ & \\
\hline \multirow{2}{*}{ Frágil } & Recuento & 3 & 49 & 52 \\
& Recuento esperado & 3,8 & 48,2 & 52,0 \\
& \% dentro de UPP & $21,4 \%$ & $27,2 \%$ & $26,8 \%$ \\
& Residuo corregido &,- 5 &, 5 & \\
\hline Prefrágil & Recuento & 0 & 33 & 33 \\
& Recuento esperado & 2,4 & 30,6 & 33,0 \\
& \% dentro de UPP & $0,0 \%$ & $18,3 \%$ & $17,0 \%$ \\
& Residuo corregido & $-1,8$ & 1,8 & \\
\hline \multirow{2}{*}{ Robusto } & Recuento & 0 & 18 & 18 \\
& Recuento esperado & 1,3 & 16,7 & 18,0 \\
& \% dentro de UPP & $0,0 \%$ & $10,0 \%$ & $9,3 \%$ \\
& Residuo corregido & $-1,2$ & 1,2 & \\
\hline Total & Recuento & 14 & 180 & 194 \\
& Recuento esperado & 14,0 & 180,0 & 194,0 \\
& \% dentro de UPP & $100,0 \%$ & $100,0 \%$ & $100,0 \%$ \\
\hline
\end{tabular}

No existe relación significativa entre los grupos de fragilidad establecidos por el SPPB y la presencia de UPP $\left(x^{2}(3)=7,36, p=, 061\right)$; aunque en el grupo de personas dependientes, el residuo corregido $(2,5)$ muestra que hay más personas con UPP de lo esperado.

En lo referente al riesgo de UPP, sí que aparecen diferencias significativas entre grupos $(F(3,187)=62,486$, $p=001$ ), de forma que aparecen dichas diferencias en todos los grupos establecidos para el SPPB (,060 > p > ,001), excepto en la relación prefrágil-robusto (tabla 2). Además, existe una correlación positiva entre el riesgo de UPP y las puntuaciones obtenidas en el SPPB total y en cada una de sus subescalas (tabla 3). En la escala Norton de riesgo de UPP, dicho riesgo aumenta a medida que la puntuación en la escala disminuye, por Io tanto, la correlación aquí obtenida indica que a mayor riesgo de escaras menor puntuación en el SPPB o todas sus subescalas; es decir, mayor es la situación de fragilidad o de dependencia. 


\section{FRAGILIDAD Y ÚLCERAS POR PRESIÓN EN PERSONAS MAYORES INSTITUCIONALIZADAS}

Tabla 2. Pruebas post hoc. Comparaciones múltiples. Bonferroni. Fragilidad-riesgo de UPP

\begin{tabular}{|c|c|c|c|c|c|c|}
\hline \multirow[t]{2}{*}{$\begin{array}{l}\text { (I) Rangos } \\
\text { SPPB }\end{array}$} & \multirow[t]{2}{*}{$\begin{array}{l}\text { (J) Rangos } \\
\text { SPPB }\end{array}$} & \multirow{2}{*}{$\begin{array}{l}\text { Diferenc } \\
\text { ia de } \\
\text { medias } \\
\text { (I-J) }\end{array}$} & \multirow{2}{*}{$\begin{array}{l}\text { Error } \\
\text { estánda } \\
\text { r }\end{array}$} & \multirow[t]{2}{*}{ Sig. } & \multicolumn{2}{|c|}{$\begin{array}{c}\text { Intervalo de } \\
\text { confianza al } 95 \%\end{array}$} \\
\hline & & & & & $\begin{array}{l}\text { Límite } \\
\text { inferior }\end{array}$ & $\begin{array}{l}\text { Límite } \\
\text { superior }\end{array}$ \\
\hline \multirow[t]{3}{*}{ Dependiente } & Frágil & $-3,342^{*}$ & ,359 & ,000 & $-4,30$ & $-2,38$ \\
\hline & Prefrágil & $-4,547^{*}$ & ,422 &, 000 & $-5,67$ & $-3,42$ \\
\hline & Robusto & $-4,689^{*}$ &, 529 &, 000 & $-6,10$ & $-3,28$ \\
\hline \multirow[t]{2}{*}{ Frágil } & $\begin{array}{l}\text { Dependient } \\
\mathrm{e}\end{array}$ & $3,342^{*}$ & 359 & ,000 & 2,38 & 4,30 \\
\hline & Prefrágil & $-1,204$ & ,462 &, 060 & $-2,44$ &, 03 \\
\hline \multirow[t]{2}{*}{ Prefrágil } & $\begin{array}{l}\text { Dependient } \\
\mathrm{e}\end{array}$ & $4,547^{*}$ & ,422 &, 000 & 3,42 & 5,67 \\
\hline & Frágil & 1,204 & ,462 &, 060 &,- 03 & 2,44 \\
\hline Robusto & $\begin{array}{l}\text { Dependient } \\
\mathrm{e}\end{array}$ & $4,689^{*}$ &, 529 & ,000 & 3,28 & 6,10 \\
\hline
\end{tabular}

Tabla 3. Rho de Spearman. Fragilidad-riesgo de UPP

\begin{tabular}{|c|c|c|c|c|c|c|}
\hline & & & $\begin{array}{l}\text { SPPB } \\
\text { equilibri } \\
\text { o }\end{array}$ & $\begin{array}{l}\text { SPPB } \\
\text { velocida } \\
\text { d }\end{array}$ & $\begin{array}{l}\text { SPPB } \\
\text { levantar } \\
\text { se }\end{array}$ & $\begin{array}{r}\text { SPPB } \\
\text { total }\end{array}$ \\
\hline \multirow[t]{3}{*}{$\begin{array}{l}\text { Rho de } \\
\text { Spearma } \\
\mathrm{n}\end{array}$} & Norton & $\begin{array}{l}\text { Coeficiente } \\
\text { de } \\
\text { correlación }\end{array}$ & $683^{* *}$ &, $718^{* *}$ & $667^{* *}$ &, $756^{* *}$ \\
\hline & & $\begin{array}{l}\text { Sig. } \\
\text { (bilateral) }\end{array}$ & ,000 &, 000 & ,000 & ,000 \\
\hline & & $\mathrm{N}$ & 192 & 192 & 191 & 191 \\
\hline
\end{tabular}

** La correlación es significativa en el nivel 0,01 (bilateral)

\section{DISCUSIÓN}

La muestra utilizada no se puede considerar representativa del total de la población de personas mayores institucionalizadas ya que se trata de un procedimiento de muestreo no probabilístico y de conveniencia que no conlleva ningún procedimiento de aleatorización que, además, implica que la validez externa puede verse comprometida. No obstante, en ciencias de la salud y sociales se trata de un tipo de muestreo ampliamente utilizado.

En la muestra que se analiza no aparece ninguna persona con UPP en los grupos de personas prefrágiles y robustas, de forma que la totalidad de las UPP registradas se distribuyen entre las personas dependientes prioritariamente $(78,6 \%)$ y las personas frágiles. Este estudio no muestra diferencias entre los grupos de fragilidad en relación a la existencia o no de UPP. Sin embargo, cuando se valora el riesgo de tener UPP aparecen diferencias estadísticamente significativas entre todos los grupos excepto entre prefrágil y robusto. Además, a mayor riesgo de UPP, mayor es la situación de fragilidad o dependencia y peores son también las puntuaciones obtenidas en las subescalas equilibrio, velocidad y levantarse. 
Los resultados de este estudio afirman que existe una relación entre la presencia de UPP y el riesgo de UPP de una parte y la fragilidad de otra. Asimismo, otros estudios han concluído que el riesgo de UPP constituye un predictor de fragilidad en el anciano (Carlos-Gil, Martínez-Pecino, Molina-Linde, Villagas-Portero, RomeroTabares, Aguilar-García, García-Alegría, Formiga y Flores-Moreno; 2011)

Dado que el principal factor de riesgo de la fragilidad es la inactividad y que la misma también supone un factor de riesgo muy importante para las UPP, las actividades centradas en la actividad física son eficaces para retrasar y revertir la fragilidad y prevenir la aparición de UPP (Da Silva González y Da Silva Domingo, 2017).

Como futuras líneas de investigación, queda abierta la posibilidad de ampliar la muestra en un estudio similar para continuar estudiando las características de las personas en situación de fragilidad que viven institucionalizadas, añadiendo más centros residenciales interesados en participar y ampliando también la distribución geográfica.

Otras líneas de investigación que pueden derivar de este estudio son las relativas a las intervenciones que de él pueden derivarse. En relación a ello se proponen intervenciones dirigidas hacia la actividad física, la nutrición, el entrenamiento o reentrenamiento en AVD, el análisis y adaptación de las actividades y del entorno a las capacidades de las personas, la revisión de la medicación, la estimulación cognitiva, programas de prevención de caídas y de UPP.

\section{CONCLUSIONES}

A la luz de los resultados, se puede concluir que el cribado de fragilidad realizado con el SPPB establece diferencias en cuanto al riesgo de caídas entre las personas frágiles, las robustas y las dependientes, pero no con el grupo de personas prefrágiles que, aunque muestran diferencias significativas con el grupo dependiente, no lo hacen con robusto y frágil, que son los grupos contiguos en el mencionado continuum.

Los resultados obtenidos permiten la detección de la población con mayor riesgo de UPP, lo que posibilitaría implementar intervenciones precoces encaminadas a la prevención de las mismas en base a las diferencias encontradas entre los grupos.

Las intervenciones deben implementarse de manera interdisciplinar, de forma que todos los profesionales que trabajen con este colectivo lo hagan en una misma dirección encaminados hacia la consecución de unos mismos objetivos:

- Prevención de la fragilidad.

- Reversión de la fragilidad.

- Prevención de la dependencia y de otros efectos adversos, incluso la muerte.

Todo ello para conseguir que las personas mayores envejezcan de la mejor manera posible manteniendo su independencia y una buena calidad de vida.

\section{REFERENCIAS BIBLIOGRÁFICAS}

Abizanda Soler, P. y Rodríguez Mañas, L. (2014). Evolución histórica del término "fragilidad". Realidad actual. En S. E. Gerontología, Guía de buena práctica clínica en geratría. Fragilidad y nutricion en el anciano. Madrid, España: Coordinación editorial: International Marketing and Communication.

Cabrero García, J., Cabañero Martínez, M. J., Ramos Pichardo, J. D. y Reig Ferrer, A. (2012). Valores de referencia de la Short Physical Performance Battery para pacientes de 70 y más años en atención primaria de salud. Atención Primaria, 44(9), 540-548.

Carlos-Gil, A. M., Martínez-Pecino, F., Molina-Linde, J. M., Villegas-Portero, R., Romero-Tabares, A., AguilarGarcía, J., ... \& Flores-Moreno, S. (2011). Predictores de fragilidad en el anciano. Rev. multidiscip. gerontol, 67-73.

Da Silva González, Á., y Da Silva Domingo, G. (2017). La fragilidad. (I. R. Psicología, Ed.) International Journal of Developmental and Educational Psychology, 1(2), 13-32. 


\section{FRAGILIDAD Y ÚLCERAS POR PRESIÓN EN PERSONAS MAYORES INSTITUCIONALIZADAS}

Fried, L., Tangen, C., Walston, J., Newman, A., Hirch, A., Gottdiener, J., . . McBurnie, M. (2001). Frailty in older adults: evidence for a phenotype. The Journals or Gerontology Series A: Biological Sciences and Medical Sciences, 56A(3), M146-M156.

García Fernández, F. P., Pancorbo Hidalgo, P. I., Soldevilla Ágreda, J. J. y Blasco García, C. (2008). Escalas de valoración del riesgo de desarrollar úlceras por presión. Gerokomos, 19(3), 136-144.

Gómez, J. F., Curcio, C. L., Alvarado, B., Zunzunegui, M. V. y Guralnik, J. (2013). Validity and reliability of the Short Physical Performance Battery (SPPB): a pilot study on mobility in the Colombian Andes. Colombia Médica, 44(3), 165-171.

González-Ruiz, J. M., Sebastián-Viana, T., Losa-Iglesias, M. E., Lema-Lorenzo, I., Crespo, F., Martín-Merino, G., ... Nogueiras-Quintas, C. G. (2014). Braden Scale and Norton Scale modified by INSALUD in acute care hospital: validity and cutoff point. Advances in skin \& wound care, 27(11), 506-511.

Ministerio de Sanidad, Servicios Sociales e Igualdad. (2014). Documento consenso sobre fragilidad y caídas. Estrategia de promoción de la Salud y Prevención en el SNS. (S. S. Ministerio de Sanidad, Ed.) Madrid, España.

Miralles Basseda, R. y Esperanza Sanjuán, A. (2006). Instrumentos y escalas de valoración. En Sociedad Española de Gerontología (coord.), Tratado de geriatría para residentes (págs. 771-789). Madrid, España: International Marketing and Communication.

Pavasini, R., Guralnik, J., Brown, J. C., di Bari, M., Cesari, M., Landi, F., ... Campo, G. (2016). Short Physical Performance Battery and all-cause mortality: systematic review and meta-analysis. BMC Medicine, 14(1), 215.

Poveda Asensio, V. (2014/2015). Recopilación de test de campo para la valoración de la condición física en mayores (trabajo final de grado). Universidad Miguel Hernández. Recuperado de: $\quad$ https://pdfs.semanticscholar.org/170c/416cce7a2dbb4b76164e7b2aafa76f1dfeb6.pdf

Proyecto ViviFrail. (s.f.). www.vivifrail.com. Recuperado de: http://www.vivifrail.com/es

Romanos Calvo, B. y Casanova Cartié, N. (2017). Norton scale modified by INSALUD and its differences in clinical practice. Gerokomos, 28(4), 194-199. 